\title{
The relationships between the isoelectric point and: length of proteins, taxonomy and ecology of organisms
} Joanna Kiraga ${ }^{1}$, Pawel Mackiewicz ${ }^{1}$, Dorota Mackiewicz ${ }^{1}$, Maria Kowalczuk1, Przemysław Biecek ${ }^{1}$, Natalia Polak ${ }^{1}$, Kamila Smolarczyk ${ }^{1}$, Miroslaw R Dudek ${ }^{2}$ and Stanislaw Cebrat*1

Address: ${ }^{1}$ Department of Genomics, University of Wrocław, ul. Przybyszewskiego 63/77, 51-148 Wrocław, Poland and ${ }^{2}$ Institute of Physics, University of Zielona Góra, ul. Szafrana 4A, 65-516 Zielona Góra, Poland

Email: Joanna Kiraga - aika@microb.uni.wroc.pl; Pawel Mackiewicz - pamac@microb.uni.wroc.pl; Dorota Mackiewicz - dorota.s@microb.uni.wroc.pl; Maria Kowalczuk - kowal@microb.uni.wroc.pl; Przemysław Biecek - Przemyslaw.Biecek@pwr.wroc.pl; Natalia Polak - nawojka@microb.uni.wroc.pl; Kamila Smolarczyk - kamila@microb.uni.wroc.pl; Miroslaw R Dudek - mdudek@proton.if.uz.zgora.pl; Stanislaw Cebrat* - cebrat@microb.uni.wroc.pl

* Corresponding author

Published: 12 June 2007

BMC Genomics 2007, 8:163 doi:10.1/86/147|-2164-8-163
Received: 7 September 2006

Accepted: 12 June 2007

This article is available from: http://www.biomedcentral.com/I47I-2/64/8/163

(c) 2007 Kiraga et al; licensee BioMed Central Ltd.

This is an Open Access article distributed under the terms of the Creative Commons Attribution License (http://creativecommons.org/licenses/by/2.0), which permits unrestricted use, distribution, and reproduction in any medium, provided the original work is properly cited.

\begin{abstract}
Background: The distribution of isoelectric point (pl) of proteins in a proteome is universal for all organisms. It is bimodal dividing the proteome into two sets of acidic and basic proteins. Different species however have different abundance of acidic and basic proteins that may be correlated with taxonomy, subcellular localization, ecological niche of organisms and proteome size.
\end{abstract}

Results: We have analysed 1784 proteomes encoded by chromosomes of Archaea, Bacteria, Eukaryota, and also mitochondria, plastids, prokaryotic plasmids, phages and viruses. We have found significant correlation in more than $95 \%$ of proteomes between the protein length and pl in proteomes - positive for acidic proteins and negative for the basic ones. Plastids, viruses and plasmids encode more basic proteomes while chromosomes of Archaea, Bacteria, Eukaryota, mitochondria and phages more acidic ones. Mitochondrial proteomes of Viridiplantae, Protista and Fungi are more basic than Metazoa. It results from the presence of basic proteins in the former proteomes and their absence from the latter ones and is related with reduction of metazoan genomes. Significant correlation was found between the pl bias of proteomes encoded by prokaryotic chromosomes and proteomes encoded by plasmids but there is no correlation between eukaryotic nuclear-coded proteomes and proteomes encoded by organelles. Detailed analyses of prokaryotic proteomes showed significant relationships between pl distribution and habitat, relation to the host cell and salinity of the environment, but no significant correlation with oxygen and temperature requirements. The salinity is positively correlated with acidicity of proteomes. Host-associated organisms and especially intracellular species have more basic proteomes than free-living ones. The higher rate of mutations accumulation in the intracellular parasites and endosymbionts is responsible for the basicity of their tiny proteomes that explains the observed positive correlation between the decrease of genome size and the increase of basicity of proteomes. The results indicate that even conserved proteins subjected to strong selectional constraints follow the global trend in the pl distribution.

Conclusion: The distribution of $\mathrm{pl}$ of proteins in proteomes shows clear relationships with length of proteins, subcellular localization, taxonomy and ecology of organisms. The distribution is also strongly affected by mutational pressure especially in intracellular organisms. 


\section{Background}

The abundance of genomic data allows for answering questions concerning the whole genomes' or proteomes' structure and evolution. One of the most intriguing recent findings is the distribution of isoelectric point (pI) of proteins in the whole proteomes. So called virtual 2D gels (i.e. plots where pI of proteins is plotted against their molecular weight) seem to be universal - in all proteomes they are usually bimodal with very low fractions of proteins with pI close to 7.4 [1-8]. More detailed analyses have shown that the distribution is connected with specific properties of basic and acidic residues of amino acids and combinations of their $\mathrm{pK}$ values. The probability of constructing a protein with pI close to 7.4 of naturally occurring amino acids is low. This is in agreement with expectations because proteins are most insoluble, least reactive and unstable in $\mathrm{pH}$ close to their $\mathrm{pI}$, and $\mathrm{pH}$ of the majority of the cell interior compartments is close to 7.5 $[5,9]$. Thus, this property of proteomes could be the result of selection at the very early steps of evolution.

Different relationships between $\mathrm{pI}$ and other phenomena were discovered. Schwartz et al. [6] observed the correlation between the trimodal distribution of $\mathrm{pI}$ and the subcellular localization of proteins. Knight et al. [7] characterized more than 100 prokaryotic and eukaryotic proteomes by virtual 2D-gels and observed very little or no relations to phylogeny while significant relationship existed with the ecological niche of organisms. Moreover, they found a negative correlation between proteome size and basicity for the smallest and the most basic proteomes. Also very well documented has been the shift of pI distribution toward acidicity in halophilic bacteria which is probably related to their adaptation to high salt environments [10-12]. Similarly, it has been assumed that the basic proteomes of Coxiella burnetti and Helicobacter pylori are connected with an adaptation to acidic environment [13] and the basic proteomes of some Archaea are an adaptation to high temperature [14]. On the other hand, Nandi et al. [15] have focused on an evolutionary approach and found that the molecular weight of proteins is a much more conserved feature than their $\mathrm{pI}$ value. They concluded that a lot of orthologous proteins change their pI between acidic and basic and only a few stay exclusively acidic or basic in different organisms. Furthermore, Schwartz et al. [6] and Knight et al. [7] found that membrane proteins are larger and more basic than non-membrane ones whereas Nandi et al. [15] found that many orthologous membrane proteins have very variable $\mathrm{pI}$ values and may be used as markers to predict the organism's ecological niche.

In this paper we have broadened our analysis to proteomes of organelles, viruses and bacteriophages and applied different methods and parameters to describe quantitatively the pI distribution of proteomes. One of the aims of these studies is uncovering the relationship between the $\mathrm{pI}$ and the protein length. In the previous analyses, such correlations even if existing, were neglected. Furthermore, we have analysed the relations between the pI distributions of proteomes and the taxonomy and ecology of corresponding organisms considering different taxonomical levels and ecological signatures such as habitat, relation to the host cell, salinity, oxygen and temperature requirements. Moreover, we have tried to explain the observed relationship between the proteome size and the pI distribution of proteomes [7].

\section{Results and Discussion General properties of isoelectric point distributions in proteomes}

Some examples of the distributions of isoelectric points of proteins coded by selected proteomes are shown in Fig. 1 (for more proteomes see additional data files 2, 3 and 4). All analysed proteomes show usually bimodal distributions with a smaller third peak between two main peaks, which are in agreement with the results of other authors $[7,8]$ and suggestions that the multimodal distribution of $\mathrm{pI}$ corresponds to the $\mathrm{pK}$ values of amino acid moieties $[8,14]$.

To simplify comparative analyses of the pI distribution of different proteomes, we have divided the whole sets of proteins into two sets called later the acidic and basic sets. The division point corresponds to the pI value for which the $\mathrm{pI}$ distribution reaches the minimum between acidic and basic sets (see the Methods section for details). For most of proteomes the division point was between 7.4 and 7.5. The distribution of the values of division points is very narrow - the range is $7.22-7.54$ with the average and median 7.41. We have not found any correlation between the $\mathrm{pH}$ of these points and the taxonomical or ecological classification of organisms and genome sizes. The universality of the $\mathrm{pH}$ value of the minimum in pI distributions supports the conclusion of other authors that the bimodal distribution of $\mathrm{pI}$ results from intrinsic chemical properties of amino acids [8].

For each proteome we have calculated the average pI separately for the basic and the acidic sets of proteins, the average length of proteins and the pI bias (see the Methods section). The pI bias simply describes the asymmetry of the bimodal distribution of pI (Fig. 1). It ranges from $100 \%$ to $100 \%$. These two extreme values indicate that all proteins in a given proteome are acidic or basic, respectively. Value close to zero means that a given proteome has a similar fraction of acidic and basic proteins. We have found that if a proteome has more acidic proteins, the average $\mathrm{pI}$ of proteins of the acidic set is lower and the relationship is statistically significant (correlation coeffi- 

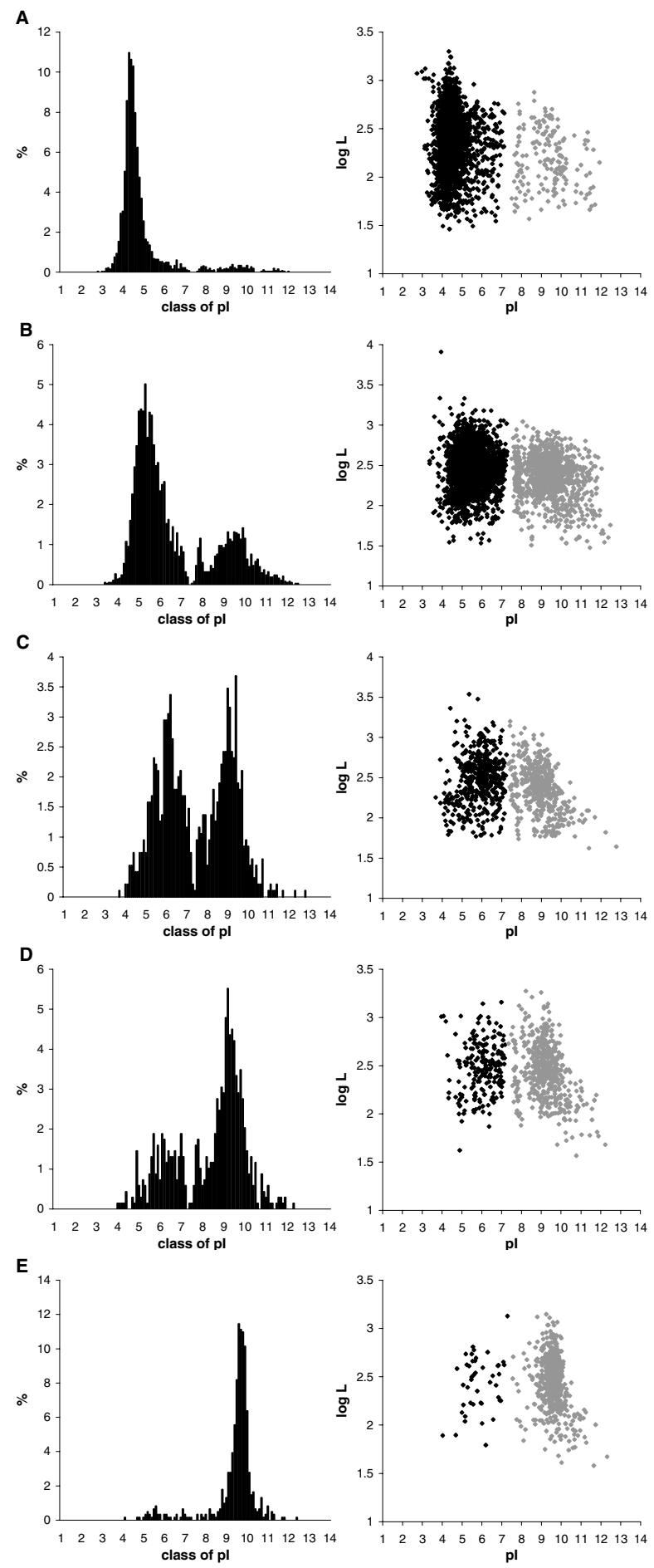

\section{Figure I}

Histograms of pl values at 0.1 unit intervals (left panel) and relationship between length of proteins (log $L$ ) and their pl (right panel) for selected prokaryotic proteomes with the different pl bias (b): (A) Natronomonas pharaonis DSM 2160, $b=-87 \%$. (B) Silicibacter pomeroyi DSS-3, b = -44\%; (C) Ehrlichia ruminantium str. Gardel, b =0\%; (D) Mycoplasma pneumoniae MI29, $b=43 \%$; (E) Wigglesworthia glossinidia, $b=86 \%$. Black points represent the set of acidic proteins while grey ones - the set of basic proteins. Diagrams for all analysed proteomes are available in additional data files 2, 3 and 4. 
cient, $\mathrm{r}=0.76, \mathrm{p}<0.001$ ) - additional data file 5A. On the other hand, if the basic proteins prevail in the proteome it is not connected with the shift of average $\mathrm{pI}$ value of basic proteins with exception of some intracellular microorganisms (additional data file 5B). We have also found that the basic sets have greater variance of their $\mathrm{pI}$ than the acidic sets that indicates greater diversity of the basic proteins.

\section{Relationships between pl value and size of proteins}

The 2D virtual distribution of proteins shows a characteristic pattern that may resemble a "butterfly" [7] or "lungs", and no correlation has been observed between $\mathrm{pI}$ and the molecular weight of proteins. Nandi et al. [15] have found that molecular weight of proteins is a much more conserved feature than their pI which would additionally suggest that there should be no correlation between $\mathrm{pI}$ and molecular weight. However, after dividing proteomes into acidic and basic sets, the correlation between the $\mathrm{pI}$ and the size of proteins for many proteomes can be seen - positive for acidic proteins and negative for basic proteins (Fig. 1). Although the correlation coefficient is not very high, for more than $92 \%$ of acidic sets and almost $99 \%$ of basic sets of chromosome-encoded proteomes of Archaea, Bacteria and Eukaryota it is statistically significant with Benjamini-Hochberg adjusted $\mathrm{p}<0.05$. Furthermore, the correlation between the size and pI of proteins is a general feature with very narrow distributions of the correlation coefficients (shown in Fig. 2 separately for acidic and basic proteins).

To show that these correlations are intrinsic properties of proteomes, we have created three artificial proteomes consisting of randomly generated proteins characterized by: (i) the average amino acid composition of E. coli proteins and the length of the real E. coli proteins; (ii) equal frequencies of amino acids and the length of the real E. coli proteins; (iii) the average amino acid composition of the E. coli proteins and the uniform length distribution in the range of the E. coli proteins. (See additional data file 6 for distributions of pI and relationships between length of proteins and their pI for these proteomes.) The generation of artificial proteomes was repeated 100 times for each set. In each case we got significant correlations between length and $\mathrm{pI}$ values (Tab. 1). Each version of artificial proteomes produced the bimodal distribution of pI and stronger correlations between the $\mathrm{pI}$ and the length of proteins than in the real E. coli proteome. The pI bias of the real proteome does not fall within the range of pI values of generated proteomes and is nearest to the generated proteomes assuming the average amino acid composition and the length of the real E. coli proteins. The pI bias was more balanced when proteins were generated from the uniform amino acids distribution, however still without proteins with $\mathrm{pI}$ close to 7.4. In addition, the proteome became more acidic when the length of proteins corresponded to

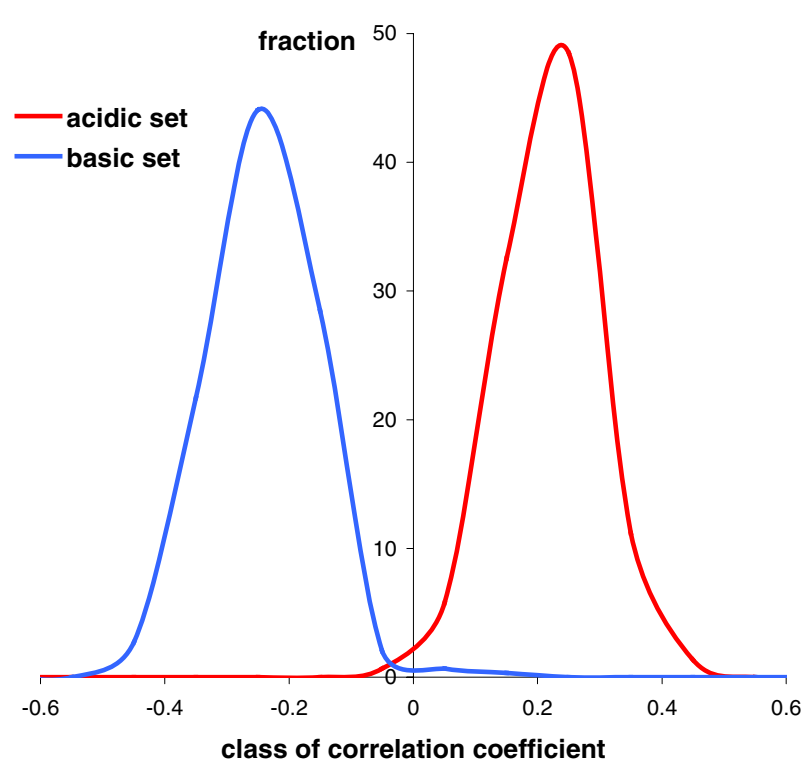

\section{Figure 2}

Distributions of the correlation coefficients between pl value and length of proteins calculated separately for acidic and basic sets of proteomes.

the uniform distribution. It suggests that selection for the length of proteins may shape the pI distribution to some extent.

The results mean that the longer proteins can maintain the neutral or nearly neutral $\mathrm{pI}$ whereas the most extreme pI values are specific for shorter proteins. It probably results from purely statistical reasons. The shorter proteins show higher fluctuation of amino acid composition, which strongly influences their pI - incorporation of even one charged residue shifts significantly the protein $\mathrm{pI}$ to the lower or higher value. On the other hand, long proteins, usually composed of more charged amino acids much better buffer the effect of fluctuation in their composition and can keep their $\mathrm{pI}$ even close to 7.4. This 'statistical' explanation of the relationship between proteins size and pI does not exclude the possibility that this relation may have some biological consequences in a diversification of proteins' structure and function. For example, if there exists a selection constraint to generate a very acidic or very basic protein, the easiest way to accomplish that is "to make" it short. Actually, very basic proteins interacting with nucleic acids - ribosomal proteins - are usually very short. We think that more proteins subjected to such selection should be found. These proteins may belong to specific regulatory proteins, transcriptional factors, modulators, signalling proteins, small proteins interacting with other proteins etc. 
Table I: Properties of artificial and real E. coli proteomes.

\begin{tabular}{|c|c|c|c|}
\hline \multirow[t]{2}{*}{ Analysed proteome } & \multicolumn{2}{|c|}{ Correlation coefficient for } & \multirow[t]{2}{*}{ pl bias [\%] } \\
\hline & acidic set & basic set & \\
\hline $\begin{array}{l}\text { the average amino acid composition of } E \text {. coli proteins; } \\
\text { the length of } E \text {. coli proteins }\end{array}$ & 0.23 & -0.28 & $-32(-29$ to -36$)$ \\
\hline $\begin{array}{l}\text { equal frequencies of amino acids; } \\
\text { the length of } E \text {. coli proteins }\end{array}$ & 0.32 & -0.28 & $-5(-8$ to -2$)$ \\
\hline $\begin{array}{l}\text { the average amino acid composition of } E \text {. coli proteins; } \\
\text { the uniform length distribution in the range of } E \text {. coli proteins }\end{array}$ & 0.33 & -0.48 & $-50(-53$ to -46$)$ \\
\hline the real proteome of $E$. coli $\mathrm{K} / 2$ & 0.15 & -0.25 & -27 \\
\hline
\end{tabular}

Each of the first three proteomes was generated 100 times and the resulting correlation coefficients and the pl bias were averaged over 100 values. Correlation coefficients both for all generated artificial proteomes and the real proteome are statistically significant with $p<0.001$.

We have also noticed a difference in the length of acidic and basic proteins. The comparative analyses have shown that acidic proteins are significantly longer than basic ones (t-Student test, Benjamini-Hochberg adjusted $\mathrm{p}<$ 0.05 ) in more than 95\% of Archaea, Bacteria and Eukaryota proteomes. The acidic proteins are on average 73 and 107 amino acid residues longer than the basic ones in the case of Prokaryota and Eukaryota, respectively. Probably it is connected with the presence of very short ribosomal proteins in the basic set and long aminoacyl-tRNA synthetases in the acidic one [15].

Nandi et al. [15] observed that the size of orthologous proteins found in closely related organisms is much more conserved than their pI. We compared the length of orthologous proteins of many proteomes whose pI values were changed from acidic to basic or vice versa but we did not observe any statistically significant differences in the length of these proteins, either. We found that the change of pI did not depend on the length of the compared orthologs.

\section{Relationships between pl values of proteomes and taxonomy}

In the analysis of potential relationships between pI of proteomes and taxonomy we have used the $\mathrm{pI}$ bias that is quite concise but a very informative parameter emphasising the most distinct and basic differences in the pI distributions. Fig. 3 shows the result of statistical analysis of the pI bias of different groups of proteomes and the UPGMA dendrogram classifying the different groups of proteomes according to the median of the pI bias. The analysis allows for dividing proteomes into at least two groups: (1) the more basic proteomes coded by plastids, viruses and plasmids and (2) the more acidic proteomes of Archaea, Bacteria, Eukaryota, mitochondria and phages. This division is supported by $95 \%$ (subsampling method), WLS-LRT and F-test (both $\mathrm{p}<0.001$ ). Moreover, every comparison between members classified to these different groups made by Kruskal-Wallis test is statistically significant ( $\mathrm{p}<$ 0.002 ). Within the acidic group it is possible to recognize some subgroups according to the high percentage support: Eukaryota with mitochondria and Bacteria with phages. The clade containing Prokaryota and phages is rather weakly supported by subsampling analysis but it is very significant according to WLS-LRT and F-test (for both $\mathrm{p}<0.001$ ). The observed basicity of plastid, viral and plasmid proteomes is probably connected with the prevalence of basic proteins (e.g. ribosomal in the case of plastids and basic proteins interacting with nucleic acids in the case of viruses) and it may also result from higher rate of accumulation of mutations, especially in viruses and plasmids (see the section 'Relation between pI value of proteomes, their sizes and GC content of genomes' for similar a explanation for intracellular bacteria).

Our analyses of prokaryotic proteomes performed on the lower taxonomical level did not show any relation between the pI bias and phylogeny, which is in agreement with the results of Knight et al. [7]. Many monophyletic or closely related groups (e.g. taxa of Archaea or proteobacteria) were not grouped when the pI bias was used as a criterion. It indicates that the pI distribution has not been conserved during evolution of prokaryotes. We did not observe such a relationship for eukaryotic proteomes, either.

On the other hand, we have found an interesting grouping related to a phylogenetic signal when analysing mitochondrial proteomes, which are much smaller and more conserved according to their protein content than large chromosome-encoded proteomes (Fig. 4, see also Tab. 2). The analysed groups of proteomes clearly divide into two subgroups: Viridiplantae+Protista and Fungi+Metazoa. This division is strongly supported in 100\%, WLS-LRT and F-test (for both $\mathrm{p}<0.001$ ). It is in agreement with the opinion that the clade of Fungi+Metazoa called Opisthokonta is a well-established group, which is supported by many molecular data analyses [16,17]. Furthermore, the two subgroups of Metazoa: Chordata and nonChordata (including the most of phyla of invertebrates) form a well-supported clade (97\%; WLS-LRT and F-test: 


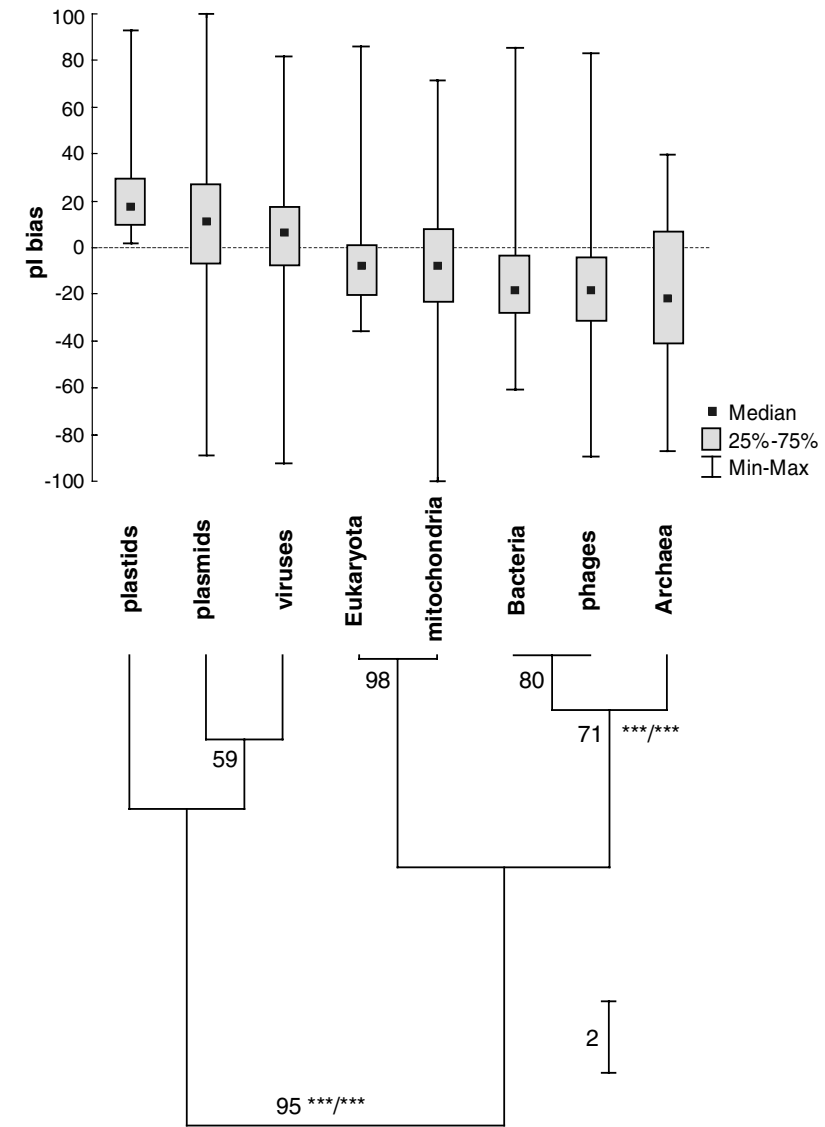

Figure 3

Statistical analysis of the pl bias of different groups of proteomes and their UPGMA-based clustering according to the median of the pl bias. Numbers at nodes mean the percentage support based on subsampling method and asterisks denote results of WLS-LRT/F tests (both with $\mathrm{p}<0.00 \mathrm{I}$ ).

for both $\mathrm{p}<0.001)$. The Viridiplantae+Protista group has much more basic proteomes on average than the Fungi+Metazoa. In the Opisthokonta group Fungi have moderately basic proteomes while Metazoa possess neutral (non-Chordata) or slightly acidic (Chordata) ones.

The observed phylogenetic signal in the $\mathrm{pI}$ bias is probably the result of different composition of proteomes. Mitochondrial proteomes of Viridiplantae, Protista and Fungi are usually several times larger than Metazoa proteomes that contain usually $12-13$ proteins. Actually, the most of proteins (e.g. ribosomal proteins) that are absent from Metazoa but present in Viridiplantae, Protista and Fungi are very basic (additional data file 7). Therefore, when we performed the same analysis based on the proteomes consisting only of 12 proteins present in the most of mitochondrial proteomes the phylogenetic signal disappeared and the pI bias of basic proteomes became neu-

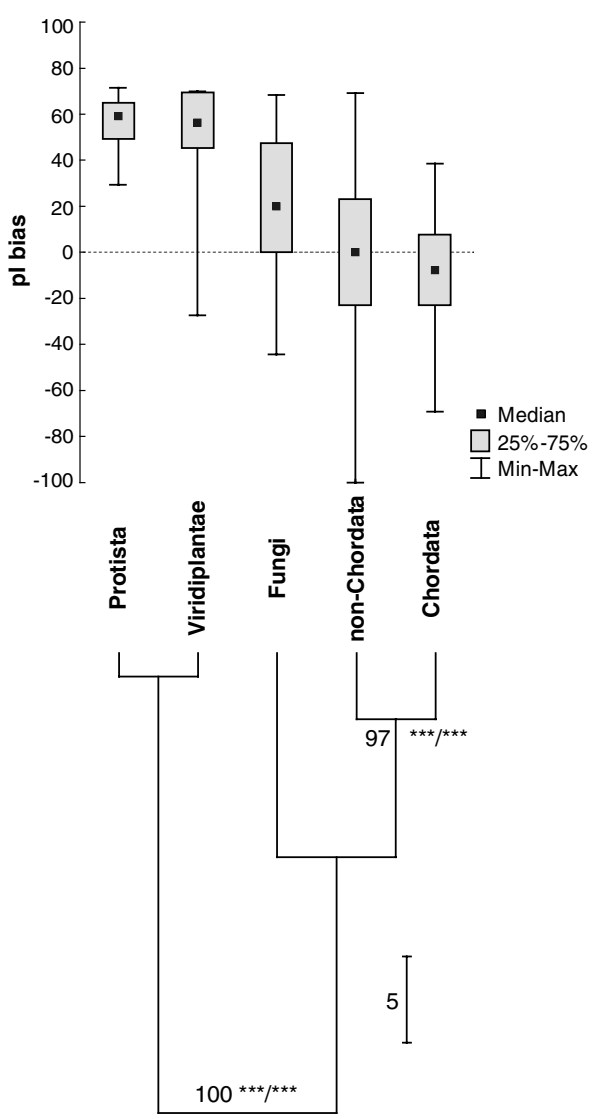

Figure 4

Statistical analysis of the pl bias of mitochondrial proteomes and their UPGMA-based clustering according to the median of the pl bias. Numbers at nodes mean the percentage support based on subsampling method and asterisks denote results of WLS-LRT/F tests (both with $p<0.00 I$ ).

tral or even acidic (for Fungi). We did not find any relationship between the pI bias and phylogeny on lower taxonomic levels: among metazoan phyla and subgroups of Craniata when analysing both full proteomes and proteomes containing only the 12 common proteins.

Similarly to Knight et al. [7] but analysing a larger set of proteomes, we have found significant correlation between the $\mathrm{pI}$ bias of proteomes coded by prokaryotic genomes and their plasmids $(\mathrm{N}=63, \mathrm{r}=0.74, \mathrm{p}<0.001)$. There is no such correlation between the pI bias of eukaryotic nuclear-coded proteomes and proteomes of their organelles $(\mathrm{N}=36, \mathrm{r}=0.04, \mathrm{p}=0.81)$. We have obtained similar results when analysing the average $\mathrm{pI}$ values of acidic and basic sets separately and no correlation was found in these two cases when the average length of proteins in these two sets was studied. It seems that organelle genomes are more independent than plasmids to follow 
Table 2: Comparison of the pl bias for subsets of organellar proteomes.

\begin{tabular}{|c|c|c|c|c|c|}
\hline \multirow[t]{2}{*}{ Analysed proteome } & \multicolumn{2}{|c|}{ Nuclear-coded proteins targeted to organelle } & \multicolumn{3}{|c|}{ Organelle-encoded proteomes } \\
\hline & the whole sequence & the mature protein & quartile $25 \%$ & median & quartile $75 \%$ \\
\hline plastid & -5.7 & -53.1 & 9.8 & 17.4 & 29.3 \\
\hline mitochondrion - Protista & 4.8 & -33.3 & 49.2 & 59.1 & 65.0 \\
\hline mitochondrion - Viridiplantae & -9.4 & -58.1 & 45.4 & 56.4 & 69.4 \\
\hline mitochondrion - Fungi & 44.4 & -6.9 & 0.0 & 20.0 & 47.4 \\
\hline mitochondrion - non-Chordata & 21.9 & -34.4 & -23.1 & 0.0 & 23.1 \\
\hline mitochondrion - Chordata & 46.0 & -15.0 & -23.1 & -7.7 & 7.7 \\
\hline
\end{tabular}

the genomic trends in pI, which seems to be not true for plasmids. For example, the organelles are separated by two membranes and posses their own replicational, transcriptional and translational machinery. Plasmids code generally for more basic proteins than chromosomes but a mutational pressure or some selection constraints affect these two proteomes simultaneously and gene transfer between plasmids and chromosomes occurs probably more often than between organellar and nuclear genomes. If more data are available, it would be interesting to analyze such relationships between viral or phagal proteomes and proteomes of infected organisms.

\section{Relationships between pl values and subcellular localization of proteins}

The analysed mitochondrial and plastid proteomes include only proteins coded by the organellar genomes. However, there are many proteins encoded by nuclear genes, which are targeted to the organelles. The nuclearencoded organellar proteins are usually equipped with $\mathrm{N}$ terminal targeting signals (transit peptides) responsible for their import into organelles. These peptides are cleaved off by organellar peptidases after the proteins are transported. Because these presequences are rich in basic amino acid residues, the pI of premature unprocessed proteins (i.e. the whole sequences) should be more basic than the $\mathrm{pI}$ of mature proteins (i.e. without transit peptide). In the Tab. 2 we have compared the pI bias of the premature and mature proteins with proteomes coded by organellar genomes. Because mitochondrial proteomes differ between various taxonomical groups, we analysed them separately. (See additional data file 8 for distributions of pI and relationships between length of proteins and their pI for these proteomes.) As it was expected, the presence of very basic transit peptides (pI bias $93-100 \%$ ) in the premature proteins shifts the pI distribution of these proteins towards basicity (i.e. higher values of pI bias), whereas most mature proteins are acidic. Interestingly, only in plastid and mitochondrial proteomes of plants a weak surplus of acidic premature proteins still exists. The nuclear-encoded organellar proteomes generally differ from the organelle-encoded ones. The pI bias values only of the mitochondrial premature proteins of Viridiplantae and Fungi and mitochondrial mature proteins of Chordata fall within the quartile range of the pI bias of corresponding organelle-encoded proteomes.

Schwartz et al. [6] analyzed three subcellular localizations of proteins and found that cytoplasmic and integral membrane proteomes contain more acidic and basic proteins, respectively, whereas the nuclear proteome has rather equal proportions of the acidic and basic proteins. In this paper we have considered 12 subcellular localizations in analyses (Tab. 3). (See also additional data file 9 for distributions of pI and relationships between length of proteins and their pI for these proteomes.) The results showed that the most acidic proteomes are present not only in cytosol but also in vacuoles and lysosomes and many acidic proteins build cytoskeleton. The most basic is the mitochondrial proteome and the distribution of $\mathrm{pI}$ of proteins integral to membrane is slightly shifted towards basicity. The rest of subcellular localizations contain more balanced proportions of acidic and basic proteins. The basic character of integral membrane proteins may be explained by the presence of the basic residues on either side of membrane spanning region, which play a role in a stabilization of proteins in the membrane [6]. Basicity of mitochondrial proteins results from the presence of positively charged transit peptides. The other relationships between the pI and the subcellular localization are probably related to the presence of some specific groups of proteins in particular localizations (e.g. acid hydrolases in lysosomes, degradative enzymes in vacuoles and acidic proteins such as: actins, dyneins, keratins, kinesins, lamins, myosins, tubulins in cytoskeleton). It is interesting to correlate the pI distribution of the particular proteomes with the $\mathrm{pH}$ of the compartments in which these proteomes are located. However, we did not observe any statistically significant correlation $(\mathrm{r}=0.38, \mathrm{p}=0.27, \mathrm{n}=10)$.

Relationships between pl values of proteomes and ecology of their organisms

To analyse the relationships between the $\mathrm{pI}$ of proteomes and ecology of organisms in details, all analysed prokaryotic proteomes were classified to different ecological subgroups (see the Methods section for details) and then 
Table 3: Comparison of the pl bias for proteomes of different subcellular localization.

\begin{tabular}{lcc}
\hline Proteome & number & pl bias \\
\hline vacuolar & 39 & -69.2 \\
cytosolic & 940 & -54.5 \\
cytoskeleton & 953 & -51.7 \\
lysosomal & 143 & -45.5 \\
Golgi apparatus & 315 & -11.7 \\
nuclear & 4144 & -10.5 \\
peroxisomal & 174 & -2.3 \\
extracellular & 1364 & 4.1 \\
endoplasmic reticulum & 695 & 4.2 \\
chloroplast & 400 & 5.5 \\
integral to membrane & 2191 & 13.7 \\
mitochondrial & 1681 & 35.8 \\
\hline
\end{tabular}

compared in respect to the pI bias (Tab. 4). The KruskalWallis tests revealed that the differences were not statistically significant when organisms were grouped according to their oxygen and temperature requirements $(p>0.1)$ but they were very significant when classified according to their habitat, relation to host cell and salinity requirements $(p<0.001)$. The same conclusion we have drawn (on the same significance level) when the $\chi^{2}$ test was performed. Fig. 5 presents the ratios of the observed to expected number of proteomes in a given class of pI bias for different ecological classifications.

The analyses showed that salinity is positively correlated with the acidity of proteomes - the more halophilic organisms have more acidic proteomes. It agrees with results of other authors who observed in halophiles predominance of acidic over basic residues [18-22] and low isoelectric point of their proteins [10-12]. Extremely halophilic and moderately halophilic bacteria are present only in the 'acidic' class and mesohalophiles disappear in the 'basic' class (Fig. 5C). This relationship is usually explained by the higher stability and solubility of proteins rich in acidic residues in hypersaline environment [19,2328].

Considering habitat preferences, host-associated organisms have the least acidic proteomes compared to other groups and aquatic bacteria possess the most acidic ones. In the 'acidic' class aquatic bacteria are the most overrepresented group and host-associated species the most underrepresented one (Fig. 5D). On the other hand, the 'basic' class contains only host-associated microorganisms. Although proteomes of host-associated species are shifted towards more basic proteomes, they are still acidic on average (Tab. 4). However, a more detailed classification of organisms considering their relation to the host cell has revealed that proteomes of intracellular bacteria are on average basic and extracellular and free-living/
Table 4: Statistical analysis of the pl bias for different ecological groups.

\begin{tabular}{|c|c|c|c|c|}
\hline Ecological group & number & quartile $25 \%$ & median & quartile $75 \%$ \\
\hline \multicolumn{5}{|l|}{$\begin{array}{l}\text { oxygen } \\
\text { requirements }\end{array}$} \\
\hline anaerobes & 36 & -28.4 & -15.4 & -3.1 \\
\hline microaerophiles & 4 & -25.5 & -8.8 & 3.3 \\
\hline facultative & 96 & -26.8 & -20.7 & -13.3 \\
\hline aerobes & 96 & -29.8 & -12.6 & 9.8 \\
\hline \multicolumn{5}{|l|}{$\begin{array}{l}\text { temperature } \\
\text { requirements }\end{array}$} \\
\hline psychrophiles & 2 & -30.6 & -21.9 & -13.2 \\
\hline mesophiles & 202 & -29.6 & -19.0 & -3.3 \\
\hline thermophiles & 13 & -27.8 & -20.8 & -13.5 \\
\hline hyperthermophiles & 15 & -23.4 & 0.0 & 12.2 \\
\hline \multicolumn{5}{|l|}{ salinity } \\
\hline extreme halophiles & 3 & -84.4 & -79.5 & -60.8 \\
\hline $\begin{array}{l}\text { moderate } \\
\text { halophiles }\end{array}$ & 8 & -49.4 & -48.3 & -44.8 \\
\hline mesohalophiles & 41 & -38.7 & -23.4 & -3.4 \\
\hline non-halophiles & 180 & -24.8 & -16.3 & -1.3 \\
\hline \multicolumn{5}{|l|}{ habitat } \\
\hline aquatic & 25 & -40.4 & -32.7 & -3.4 \\
\hline multiple & 56 & -33.8 & -29.4 & -21.0 \\
\hline specialized & 22 & -48.2 & -21.2 & -9.9 \\
\hline terrestrial & 12 & -26.0 & -22.9 & -20.3 \\
\hline host-associated & 117 & -19.8 & -10.6 & 12.8 \\
\hline \multicolumn{5}{|l|}{$\begin{array}{l}\text { relation to host } \\
\text { cell }\end{array}$} \\
\hline free living & 76 & -38.4 & -25.7 & -13.6 \\
\hline $\begin{array}{l}\text { free living/ } \\
\text { extracellular }\end{array}$ & 41 & -33.7 & -26.5 & -20.5 \\
\hline $\begin{array}{l}\text { free living/ } \\
\text { intracellular }\end{array}$ & 4 & -12.9 & -10.6 & -7.8 \\
\hline extracellular & 70 & -22.1 & -17.5 & -4.7 \\
\hline intracellular & 41 & -8.8 & 14.2 & 42.8 \\
\hline
\end{tabular}

intracellular species have slightly acidic proteomes (Fig. $5 \mathrm{E})$. More acidic proteomes are characteristic of free-living/extracellular and free-living species. Actually, these two groups are overrepresented in the 'acidic' class whereas intracellular bacteria are strongly overrepresented in the 'basic' class. The results show that the more an organism is related to the host cell the more basic proteome it has. The explanation of this result will be discussed in the next section where the relationship between the pI bias, proteome size and GC content of genome is considered. We have also noticed that all intracellular organisms that have slightly acidic proteomes (Anaplasma, Brucella, Chlamydiae, Ehrlichia) and the majority of them (with only one exception) that have slightly basic proteomes with the pI bias $\leq 20 \%$ (Bartonella, Coxiella, Parachlamydia, Rickettsia conorii, Tropheryma, Wolbachia) reside and usually replicate in vacuoles or 

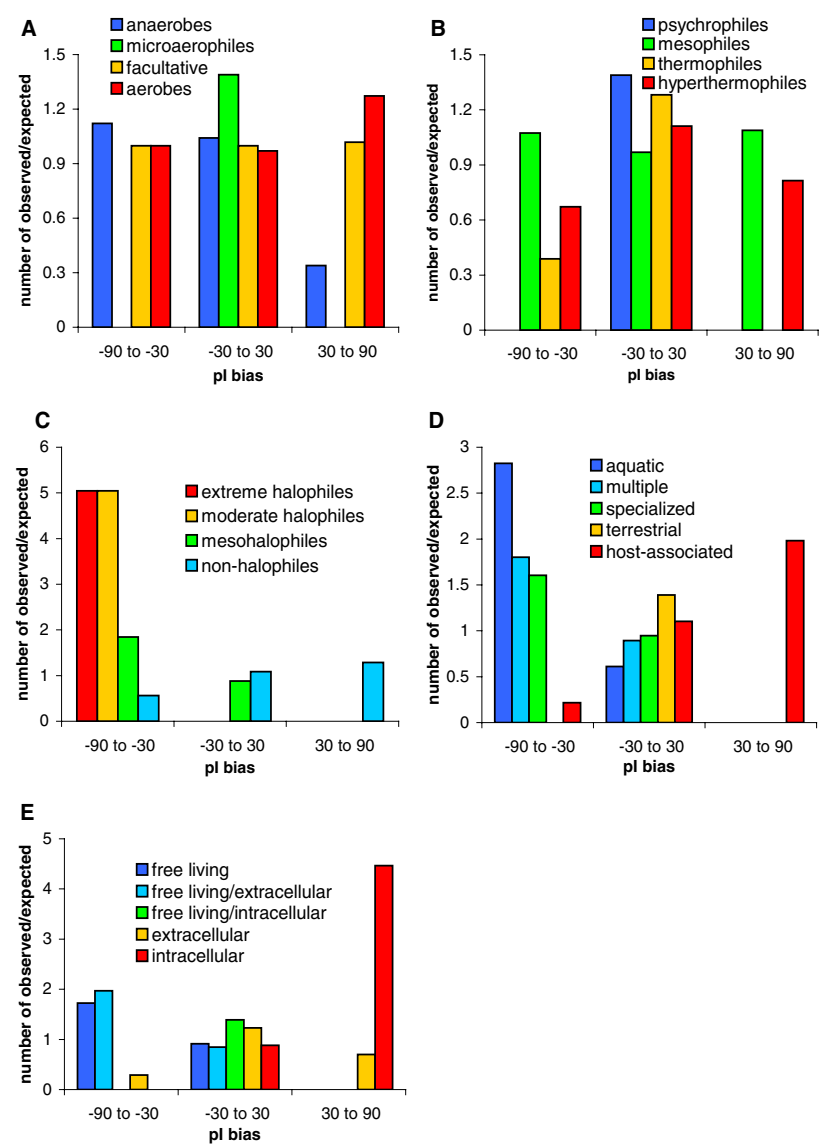

Figure 5

Ratios of the observed to expected number of proteomes in a given class of pl bias for different ecological classifications: (A) oxygen, (B) temperature, (C) salinity, (D) habitat and (E) relation to host cell.

phagosomes. What is interesting, $R$. conorii has the least basic proteome within the Rickettsia genus and as the only representative of its genus was observed in a vacuole [29]. It would imply that environment of vacuoles modifies proteomes of intracellular organisms towards acidicity.

Ecological changes quite strongly and quickly influence the pI bias in the course of evolution of proteomes because the changes in the pI bias are seen even among closely related organisms. We have gathered all analysed species belonging to the same genus and having different ecological assignments (see additional data file 10). In every case the pI bias of host-associated species is more shifted towards higher values (i.e. basicity) than the bias of species living in the multiple environments. The most pronounced examples are two species of Burkholderia of which one lives in a terrestrial habitat and posses the acidic proteome and the other one is associated with a host and has the basic proteome. The proteomes of other host-associated species, although shifted towards basicity, are still acidic probably because these species are still facultative and extracellular parasites. Moreover, the clear shift of the pI bias is visible when species living in different salinity requirements are compared. The proteomes of halophilic and mesohalophilic species have more acidic proteins than non-halophilic ones.

\section{Relationships between pl values of proteomes, their sizes and GC content of genomes}

The relationship between pI value of proteomes, their sizes and GC content of genomes was analysed by Knight et al. [7]. They concluded that there is no correlation between median pI and GC content in small basic proteomes and have opened the discussion about the reasons of basicity of tiny proteomes by giving two unverified potential explanations: selection of proteins in the proteome and selection within particular proteins. To see how general these relations are, we have performed analyses on a larger set of proteomes using the pI bias and considering the relation of organisms to host cell in more detail.

Fig. 6A presents the relationship between the pI bias and proteome size (expressed as logarithm of proteins number) for prokaryotic organisms. The plot in additional data file 11 includes also eukaryotic organisms. A clear trend is visible for prokaryotic proteomes - the smaller proteomes contain more basic proteins - whereas, the most of proteomes of Eukaryota are shifted towards larger values of proteome size. The correlation coefficient for the set containing prokaryotic proteomes is $\mathrm{r}=-0.64$ ( $\mathrm{p}$ $<0.001)$. The negative correlation has been found also for the pI bias and the GC content of genomes (for the whole set: $r=-0.49$, for prokaryotic proteomes $r=-0.50$; for both $\mathrm{p}<0.001)$. Generally, the genomes with lower GC content code for more basic proteomes. Detailed analysis of prokaryotic proteomes, considering relation between organisms and the host cell have shown that the more a bacterial strain relies on its host the higher is AT fraction in its genome and the smaller and more basic proteome it possesses (Fig. 6). Most of these organisms are intracellular parasites or endosymbionts or at least they are in some way associated with their host (extracellular). It seems that the rules are general because the proteome encoded by the nucleomorph of a eukaryote Guillardia theta [30] is also AT-rich, reduced and the most basic in the whole set. G. theta is a unicellular cryptophyte alga which is a host for another cell with reduced residual nucleus called the nucleomorph. The cell may be considered endosymbiont as well.

The observed correlations are clearer when only species with proteomes smaller than 1000 proteins are consid- 


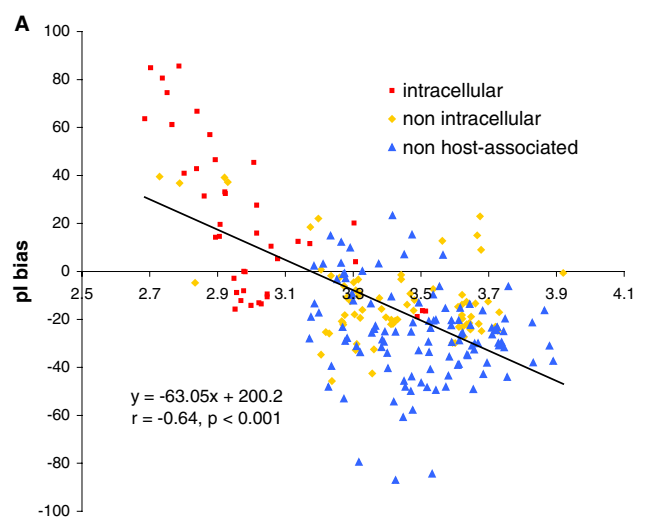

$\log$ (proteome size)

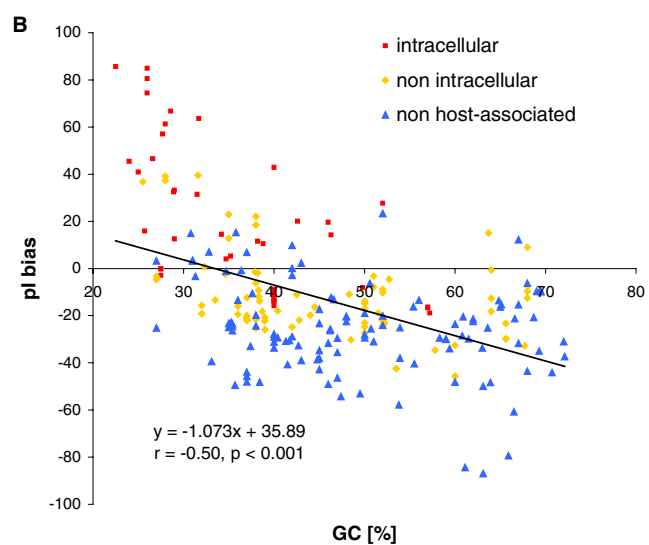

\section{Figure 6}

Relationship between the pl bias and: (A) logarithm of proteome size and (B) genomic GC content for different ecological groups of prokaryotes.

ered. The correlation coefficients change from -0.64 to 0.82 for the relationship between the pI bias and proteome size and from -0.49 to -0.58 for the relationship between the pI bias and GC content and are statistically significant. Therefore, the hypothesis that the inefficiency of DNA repairing mechanisms in intracellular microorganisms causes the large AT bias of their small genomes and in consequence greater content of basic lysine [31] can not be completely excluded. It is possible that there are some other reasons of the observed relationships between the basicity of the proteome and the reduction of its size such as: gain or loss of some groups of proteins causing the pI shift of the whole proteome or selection for pI changes of particular proteins, e.g. involved in adaptation to the new environment or host [7]. The former hypothesis explains at least the differences between mitochondrial proteomes (see above).

We have considered if this hypothesis explains the basicity of small prokaryotic proteomes as well. We can exclude acquisition of basic proteins as the explanation because it is difficult to find any new genes gained by intracellular bacteria that would be absent from the closely related freeliving relatives [32-34]. Usually, in the intracellular bacteria many genes deteriorate or are eliminated rather, leaving a conserved set of genes (e.g. encoding basic ribosomal proteins). The loss of dispensable genes coding for acidic proteins could lead to the shift of the whole proteome towards basicity. One could assume that conserved orthologous proteins present both, in the intracellular bacteria and in their free-living counterparts did not change their functions in the course of evolution and these sets have the same proportions of different classes of proteins. Then we should expect that these proteomes have similar pI distributions and are more basic than the proteins which are still present in the free-living bacteria but were lost in the intracellular organism. To check this we have analysed distribution of $\mathrm{pI}$ for three sets of proteins: present only in the free-living bacteria but not in the intracellular ones, and orthologous proteins common for the intracellular bacteria and their free-living counterparts analysed separately for these two groups of bacteria. The results of comparison of Escherichia coli K12 with: Buchnera aphidicola str. Bp, Candidatus Blochmannia floridanus and Wigglesworthia glossinidia are presented in Fig. 7. The set of orthologous proteins of these endosymbionts is very basic. However, the orthologous proteins of E. coli are even more acidic than the set of proteins present only in E. coli and lost in the endosymbionts. We have obtained similar results for seven other pairs of bacteria. Assuming that the E. coli proteome resembles the free-living ancestor of the endosymbionts we can estimate that 300 to 400 of its proteins (i.e. 55-65 percent of the common part of proteomes) have changed their pI from acidic to basic during transition to intracellular way of life. Therefore, in contradiction to the mitochondrial proteomes, the observed shift of small bacterial proteomes towards basicity cannot be explained by the gain or loss of some proteins in the proteome but by the shift of pI of many proteins.

To find the explanation of this shift, we have generated for each organism a proteome of the same length distribution of proteins as in its real proteome but with the amino acid composition calculated for the base composition characteristic for the given genome that reflects, to some extent, the composition generated by mutational pressure [35]. We generated 100 such proteomes for each organism and we averaged their $\mathrm{pI}$ bias. These proteomes show the equilibrium steady state of proteomes evolving only under mutational pressure without any selection constraints. All generated proteomes showed extremely high (higher than $85 \%$ ) pI bias with average $=94 \%$ (Fig. 8 A, see additional data file 6 for the E. coli example). Actually, according to the genetic code table, assuming equal frequencies of 

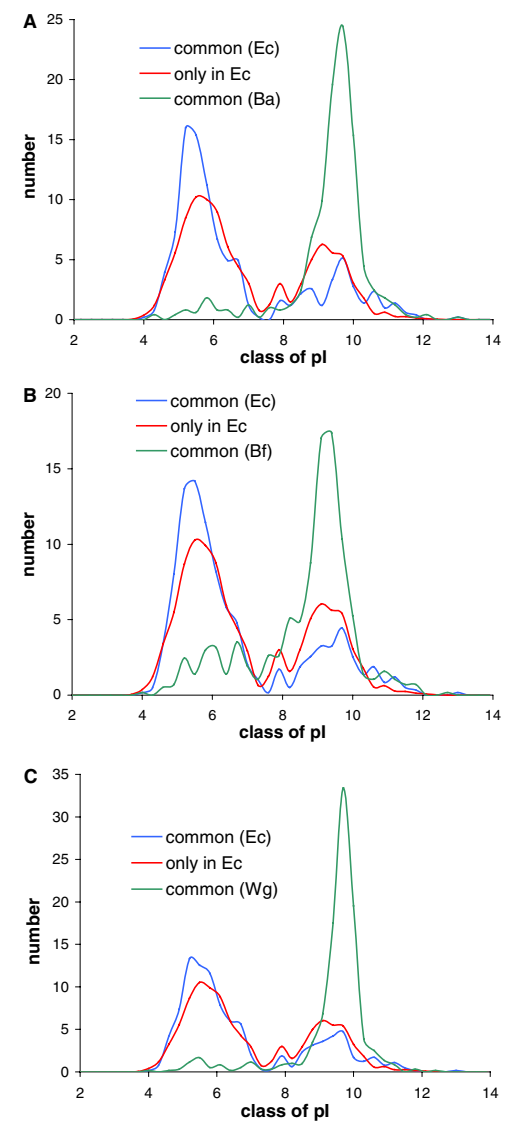

\section{Figure 7}

Distribution of pl for three sets of proteins: present in E. coli $\mathrm{K} 12$ only and not in the intracellular endosymbiont (only in Ec), E. coli proteins orthologous to the intracellular bacteria [(common $(\mathrm{Ec})]$, and the endosymbiont proteins orthologous to $E$. coli [(common (initials of endosymbiont)]. Comparisons are made for $E$. coli $\mathrm{K} I 2$ with: Buchnera aphidicola str. Bp (A), Candidatus Blochmannia floridanus (B) and Wigglesworthia glossinidia (C).

codons, representation of the acidic residues is lower than the basic ones. The relationship between the fraction of the basic or acidic amino acid and GC content is non-linear but any nucleotide composition favours basic residues especially for extreme GC content (Fig. 8B). The smallest difference between the fraction of coded basic and acidic amino acid is for $37 \%$ of GC. It is in agreement with the relationship obtained for the generated proteomes. The lowest pI bias falls between $30 \%$ and $40 \%$ of GC content. Furthermore, the points bifurcate towards the higher pI bias for the extreme values of GC content showing negative correlation below and positive correlation above $40 \%$ of GC content. The negative relationship is probably connected with the increase of basic lysine coded by AT-rich codons whereas the positive one - with the growth of basic arginine coded by GC-rich codons. Since any DNA
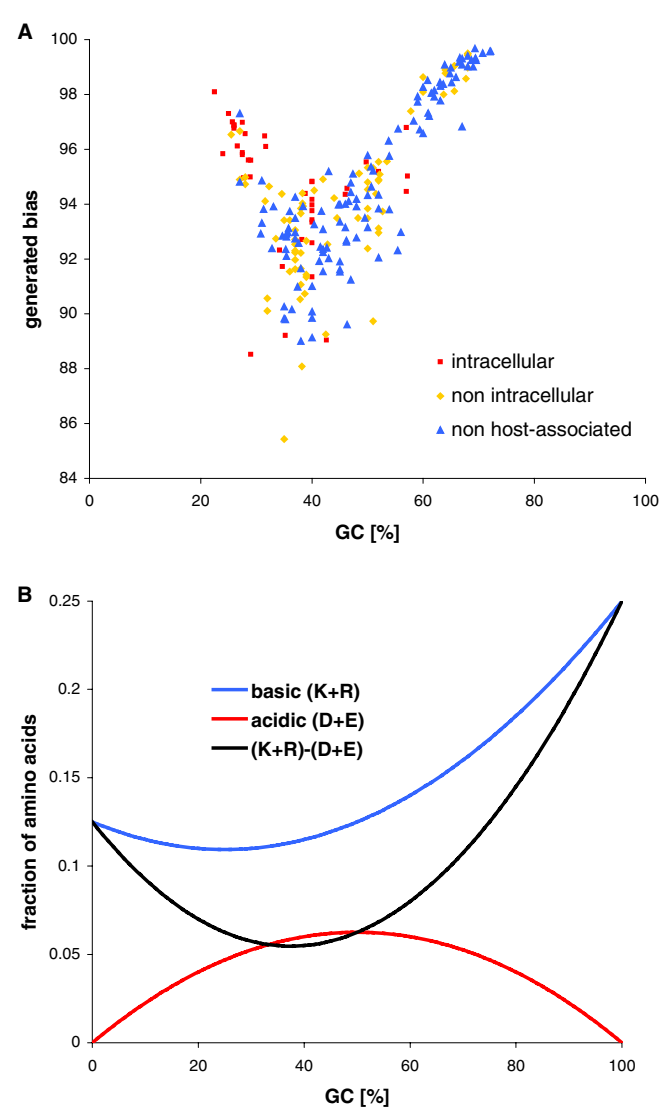

Figure 8

Isoelectric point and GC content. (A) The relationship between the computer-generated $\mathrm{pl}$ bias and the genomic GC content of prokaryotic organisms. The generated pl bias is the average calculated for 100 virtual proteomes generated for each organism assuming the same length distribution of proteins as in real proteomes and the amino acid composition calculated from the base composition characteristic for the given genome. (B) The relationship between the fraction of the basic and acidic amino acids and GC content.

composition (especially the one in equilibrium with mutational pressure) shifts proteomes towards basicity, we can assume that the acidic proteomes are subjected to strong selection pressure opposite to the mutational pressure. The more acidic a proteome is the stronger selection should be exerted on it. So far, the most acidic proteomes belong to extreme halophiles so we should expect very strong selection constraints on this group exerted by the hypersaline environment in which they live.

The results indicate that AT bias alone cannot explain the observed basicity of tiny proteomes. We have not observed any correlation for these virtual proteomes between the pI bias and proteome size also. It seems that the acceleration of mutation accumulation itself could be 
responsible for the basicity of tiny proteomes. It is in agreement with the well-documented higher evolutionary rate of intracellular bacteria than their free-living relatives. It has been suggested that the higher rate of evolution results from enhanced mutation rate [36] and/or Muller's ratchet effect or the easier fixation of mutations by genetic drift in small asexual populations [37-46]. Accordingly, the increase of basicity of proteomes and the increase of mutational AT bias would be paralelly ongoing phenomena resulting from the higher evolution rate of genomes of intracellular bacteria. The elevated AT bias probably results from the elimination of genes encoding DNA repair and recombination-associated enzymes or at least from the decrease in their efficiency in intracellular organisms $[31,32,37,47-49]$. These enzymes would normally correct the error-related tendency toward AT enrichment, for example the deamination of cytosine to uracil, which is then replaced by thymine.

To confirm our explanation we have analysed 39 sets of much conserved orthologous proteins present in each of 100 selected prokaryotic organisms, representing quite uniformly the values of the pI bias. We have assumed that if the increase in substitution rate affects the pI distribution we should observe a positive correlation between the pI of these proteins and the pI bias of the whole proteomes. Actually, for 36 of them we have found such statistically significant correlation (see additional data file 12). Three sets, for which the correlation was not significant, represent the most basic ribosomal proteins and maybe their further shift was improbable. The results indicate that even much conserved proteins subjected to strong selectional constraints follow the global trend in the pI distribution. Such a relationship concerns probably many other proteins because analyses of pI of Clusters of Orthologous Groups (COGs) showed that proteins of only few clusters are conserved and stay in the same acidic or basic set while the majority of them jump between the two sets [15]. Many of these promiscuous proteins are membrane proteins that have direct contact with the external environment and may be considered adaptive proteins. Therefore it would favour the hypothesis about the selection for pI changes of particular proteins. However, it does not fully explain the basicity of small prokaryotic proteomes because the changes of pI concern also many non-membrane and much conserved proteins. (Relationships between pI and other phenomena we discussed in additional data file 13.)

It would be interesting to investigate the relationship between the change of the pI distribution of proteomes and the transition of organisms from the free to intracellular way of life on different stages of genome reduction. Very insightful results concerning this subject would give sequencing and analysis of reduced genomes of bacterial endosymbionts identified in eukaryotic hosts, e.g. a cyanobacterium in amoeba Paulinella chromatophora, a cyanobacterium Cyanothece in diatom Rhopalodia gibba, a Gram-negative bacterium in diatom Pinnularia nobilis and many different bacterial endosymbionts found in various species of insects. Additional interesting results would come from analyses of nucleomorphs - small, reduced eukaryotic nuclei found in certain plastids present in some groups of algae such as cryptomonads and chlorarachniophytes. Moreover, it is interesting to estimate how many mutations are required or should be accepted to remodel one proteome to another in the aspect of their pI. However, many factors such as length, amino acid composition, pI of the original proteins, mutation rate and patterns of nucleotide substitutions and resulting patterns of amino acid substitutions should be taken into account.

\section{Conclusion}

Although the distribution of pI of proteins in proteomes is generally bimodal, different species have different abundance of acidic and basic proteins that is correlated with ecology of these species, especially with habitat, relation to the host cell and salinity of the environment. The pI distribution is also related with taxonomy of organism but only on higher taxonomical levels and subcellular localization of some proteomes. The other factor that shapes the distribution is the rate of mutations accumulation. The rate is higher in intracellular organisms than in free-living ones and it is responsible for the basicity of tiny proteomes that explains the observed relationship between the proteome size and pI of proteomes.

\section{Methods}

Proteomic sets were downloaded from different sources (see additional data file 1 for details): National Center for Biotechnology Information, European Bioinformatics Institute, DOE Joint Genome Institute, Broad Institute of MIT and Harvard, The Institute for Genomic Research (TIGR), Welcome Trust Sanger Institute, Ensembl project, Stanford Genomic Resources, Virginia Commonwealth University, National Institute of Genetics, Japan, PlasmoDB, International Fugu Genome Consortium, Genoscope, DictyBase and SilkDB. We have not analysed proteomes containing less than 10 proteins. In sum we have analysed 1784 proteomes grouped in the following sets encoded by: chromosomes in Archaea (22), Bacteria (210) and Eukaryota (63), mitochondria (720), plastids (42), prokaryotic plasmids (319), phages (245) and viruses (163). Note that in the paper, in the proteomes of Archaea, Bacteria and Eukaryota only chromosomeencoded proteins are referred to. Sequences of nuclearencoded proteins with annotated transit peptides and targeted to mitochondria (2021) or plastids (1173) were downloaded from UniProt database [50]. Proteomes of 12 subcellular localizations (in sum 13,039 proteins) were extracted from non-redundant datasets from DBSubLoc database [51]. 
Isoelectric points were calculated using the standard iterative algorithm [52,53] that gives relatively precise results of pI calculations for raw protein sequences $[1,2]$. The algorithm is used in the Compute $\mathrm{pI} / \mathrm{Mw}$ tool at the ExPASy server [54]. The source code of the algorithm was kindly supplied by Elisabeth Gasteiger.

Each proteome was divided into two sets named the acidic and the basic one according to the $\mathrm{pI}$ value of its proteins. To find the point of division of proteomes for the two sets, we ranked proteins according to their pI values, cut off $10 \%$ tails of both acidic and basic proteins and the rest of the proteome distribution was scanned for the largest difference in pI between two neighbouring proteins. The point of division between acidic and basic proteins was set in the middle of this distance. Because of statistical reasons, the procedure was applied only to big chromosomeencoded proteomes of Archaea, Bacteria and Eukaryota. Because of the narrow range and the universality of the mid-point, we assumed as the division point of smaller proteomes encoded by plasmids, mitochondria, plastids, viruses and phages the median of the mid point calculated for the big proteomes which equals 7.41.

Proteomes were characterized by the average $\mathrm{pI}$ and the average length of proteins separately for the basic and the acidic sets of proteins and by the "pI bias" (b) describing the relation between the number of proteins in the basic set and the number of proteins in the acidic set: $b=100$. $\left(\mathrm{N}_{\text {basic }}-\mathrm{N}_{\text {acidic }}\right) /\left(\mathrm{N}_{\text {basic }}+\mathrm{N}_{\text {acidic }}\right)$, where $\mathrm{N}_{\text {acidic }}$ and $\mathrm{N}_{\text {basic }}$ denote the numbers of proteins in the acidic and the basic sets, respectively.

The different sets of proteomes were clustered by the UPGMA (Unweighted Pair-Group Method Arithmetic Averages) method based on the median of the pI bias. The clustering was performed with the neighbour program from the PHYLIP 3.6 package [55]. To evaluate the reliability of specific clades in UPGMA trees we created 10000 matrices of median of the pI bias generated by the random sampling of 2/3 members of each group of proteomes (subsampling method). Then we applied the neighbor and consense programs (from the PHYLIP package) to calculate the percent of randomised trees containing a given clade. Moreover, the WeightLESS program [56] was used to perform the WLS-LRT (Weighted least-squares likelihood ratio test) and F-test.

All analysed prokaryotic proteomes were classified according to five ecological signatures: habitat (aquatic, host-associated, multiple, specialized, terrestrial), relation to host cell (extracellular, free living, free living/extracellular, free living/intracellular, intracellular), salinity (extreme halophilic, moderate halophilic, mesohalophilic, non-halophilic), oxygen (aerobic, anaerobic, facul- tative, microaerophilic) and temperature requirements (hyperthermophilic, mesophilic, psychrophilic, thermophilic). The classification was based on the data published on the NCBI web site, papers related to the sequenced genomes and other sources. A given species was assigned only to one subgroup, the most typical for its ecological property. To analyse the relationship between the pI bias and ecological classification of proteomes the analysed proteomes of particular ecological subgroups were distributed among three classes of the pI bias ('acidic': - $-90 \%$ to $-30 \%$, 'neutral': $-30 \%$ to $30 \%$ and 'basic': $30 \%$ to $90 \%$ ). Then the observed numbers of proteomes in the given class were compared to the expected ones by $\chi^{2}$ test.

Sets of orthologous proteins of prokaryotic organisms were downloaded from Microbial Genome Databse MBGD [57].

The non-parametric Kruskal-Wallis test and t-Student test were applied accordingly to determine statistical significance of tested hypotheses. The Benjamini-Hochberg multiple comparisons procedure for controlling the false discovery rate was used [58].

\section{Authors' contributions}

JK started this work and generated preliminary results, carried out analyses, collected data about ecological classification of organisms and wrote scripts in Perl. MP supervised the project, wrote this manuscript, downloaded the data, carried out analyses and wrote scripts in Perl. DM wrote scripts in Perl and generated sequence data. MK wrote this manuscript and contributed substantially to the final manuscript. PB performed some statistical analyses and wrote scripts in the R environment. NP and KS collected data about ecological classification of organisms and generated sequence data. MRD wrote programs in $\mathrm{C}++$ calculating isoelectric point and generating random amino acid sequences. SC drafted manuscript, provided overall guidance and interpretation of results. All authors read and approved the final manuscript.

\section{Additional material}

\section{Additional file 1}

List of analysed proteomes.

Click here for file

[http://www.biomedcentral.com/content/supplementary/1471-

2164-8-163-S1.xls] 


\section{Additional file 2}

PI distribution for prokaryotic proteomes. Left panel: histograms of pI values at 0.1 unit intervals ( $X$ axis: class of pI; $Y$ axis: percent); right panel: relationships between the logarithm of length of proteins ( $Y$ axis) and their pI (Y axis). Black points represent the set of acidic proteins while grey ones - the set of basic proteins.

Click here for file

[http://www.biomedcentral.com/content/supplementary/1471-

2164-8-163-S2.pdf]

\section{Additional file 3}

PI distribution for prokaryotic proteomes. Left panel: histograms of pI values at 0.1 unit intervals ( $X$ axis: class of pI; $Y$ axis: percent); right panel: relationships between the logarithm of length of proteins ( $Y$ axis) and their $D I$ ( $Y$ axis). Black points represent the set of acidic proteins while grey ones - the set of basic proteins.

Click here for file

[http://www.biomedcentral.com/content/supplementary/14712164-8-163-S3.pdf]

\section{Additional file 4}

PI distribution for eukaryotic proteomes. Left panel: histograms of pI values at 0.1 unit intervals ( $X$ axis: class of pI; $Y$ axis: percent); right panel: relationships between the logarithm of length of proteins ( $Y$ axis) and their pI (Y axis). Black points represent the set of acidic proteins while grey ones - the set of basic proteins.

Click here for file

[http://www.biomedcentral.com/content/supplementary/1471-

2164-8-163-S4.pdf]

\section{Additional file 5}

Relationship between the average pI of proteins and the pI bias for acidic (A) and basic sets (B).

Click here for file

[http://www.biomedcentral.com/content/supplementary/14712164-8-163-S5.pdf]

\section{Additional file 6}

The real and artificial proteomes of Escherichia coli K12. Left panel: histograms of pI values at 0.1 unit intervals ( $X$ axis: class of pI; $Y$ axis: percent); right panel: relationships between the logarithm of length of proteins ( $Y$ axis) and their pI ( $Y$ axis).

Click here for file

[http://www.biomedcentral.com/content/supplementary/14712164-8-163-S6.pdf]

\section{Additional file 7}

PI distribution of mitochondrial proteomes belonging to Fungi (A), Protista (B) and Viridiplantae (C) prepared separately for proteins common for Metazoa and the analyzed group (present in Metazoa) and absent from Metazoa.

Click here for file

[http://www.biomedcentral.com/content/supplementary/14712164-8-163-S7.pdf]

\section{Additional file 8}

PI distribution for nuclear-encoded proteins targeted to organelles: plastid and mitochondrion. Left panel: histograms of pI values at 0.1 unit intervals (X axis: class of pI; Y axis: percent); right panel: relationships between the logarithm of length of proteins ( $Y$ axis) and their pI (Y axis). Black points represent the set of acidic proteins while grey ones - the set of basic proteins. Transit peptides, premature proteins (i.e. the whole sequence) and mature proteins (i.e. without transit peptide) were analysed separately. Mitochondrial proteomes were divided to various taxonomical groups.

Click here for file

[http://www.biomedcentral.com/content/supplementary/14712164-8-163-S8.pdf]

\section{Additional file 9}

PI distribution for proteomes of different subcellular localization. Left panel: histograms of pI values at 0.1 unit intervals (X axis: class of pI; $Y$ axis: percent); right panel: relationships between the logarithm of length of proteins ( $Y$ axis) and their PI (Y axis). Black points represent the set of acidic proteins while grey ones - the set of basic proteins.

Click here for file

[http://www.biomedcentral.com/content/supplementary/1471-

2164-8-163-S9.pdf]

\section{Additional file 10}

Ecological changes and their relationships with the pI bias of proteomes in closely related organisms.

Click here for file

[http://www.biomedcentral.com/content/supplementary/14712164-8-163-S10.doc]

\section{Additional file 11}

Relationship between the pI bias and logarithm of proteome size for archaeal, bacterial and eukaryotic organisms.

Click here for file

[http://www.biomedcentral.com/content/supplementary/14712164-8-163-S11.pdf]

\section{Additional file 12}

Characteristics of 39 sets of conserved orthologous proteins present in all selected 100 prokaryotic organisms. The correlation coefficient was calculated between the pI of proteins and the pI bias of the whole proteomes. Click here for file

[http://www.biomedcentral.com/content/supplementary/14712164-8-163-S12.doc]

\section{Additional file 13}

Discussion about relationships between pI and other phenomena Click here for file

[http://www.biomedcentral.com/content/supplementary/14712164-8-163-S13.doc]

\section{Acknowledgements}

We would like to thank Elisabeth Gasteiger for sending us the source code of the algorithm calculating pl. We are grateful to two anonymous Reviewers for their insightful comments and suggestions. The work was supported by Polish Foundation for Science and by grant \#1016/S/IGiM/05. It was done in the frame of the ESF program COST Action PIO. 


\section{References}

I. Link AJ, Hays LG, Carmack EB, Yates JR 3rd: Identifying the major proteome components of Haemophilus influenzae type-strain NCTC 8 I 43. Electrophoresis 1997, I 8: | 3 |4- | 334

2. Link AJ, Robison K, Church GM: Comparing the predicted and observed properties of proteins encoded in the genome of Escherichia coli K-I2 . Electrophoresis 1997, I8: I259-I3।3.

3. VanBogelen RA, Abshire KZ, Moldover B, Olson ER, Neidhardt FC: Escherichia coli proteome analysis using the gene-protein database. Electrophoresis 1997, I8: |243-1251.

4. Urquhart BL, Cordwell SJ, Humphery-Smith J: Comparison of Predicted and Observed Properties of Proteins Encoded in the Genome of Mycobacterium tuberculosis H37Rv. Biochem Biophys Res Commun 1998, 253:70-79.

5. VanBogelen RA, Schilles EE, Thomas JD, Neidhardt FC: Diagnosis of cellular states of microbial organisms using proteomics. Electrophoresis 1999, 20:2149-2159.

6. Schwartz R, Ting CS, King J: Whole Proteome pl Values Correlate with Subcellular Localizations of Proteins for Organisms within the Three Domains of Life. Genome Res 200I, I I:703-709.

7. Knight ChG, Kassen R, Hebestreit H, Rainey PB: Global analysis of predicted proteomes: Functional adaptation of physical properties. Proc Natl Acad Sci USA 2004, I 0 I:8390-8395

8. Weiller GF, Caraux G, Sylvester N: The modal distribution of protein isoelectric points reflects amino acid properties rather than sequence evolution. Proteomics 2004, 4:943-949.

9. Arakawa T, Timasheff SE: Theory of protein solubility. Methods Enzymol 1985, I | 4:49-77.

10. Kennedy SP, Ng WV, Salzberg SL, Hood L, DasSarma S: Under standing the adaptation of Halobacterium species NRC-I to its extreme environment through computational analysis of its genome sequence. Genome Res 200 I, I I: I64 I- |650.

II. Goo YA, Roach J, Glusman G, Baliga NS, Deutsch K, Pan M, Kennedy S, DasSarma S, Ng WV, Hood L: Low-pass sequencing for microbial comparative genomics. BMC Genomics 2004, 5:3.

12. Mongodin EF, Nelson KE, Daugherty S, Deboy RT, Wister J, Khouri H, Weidman J, Walsh DA, Papke RT, Sanchez Perez G, Sharma AK Nesbo CL, MacLeod D, Bapteste E, Doolittle WF, Charlebois RL, Legault B, Rodriguez-Valera F: The genome of Salinibacter ruber : convergence and gene exchange among hyperhalophilic bacteria and archaea. Proc Natl Acad Sci USA 2005, 102:18147-18152

13. Seshadri R, Paulsen IT, Eisen JA, Read TD, Nelson KE, Nelson WC Ward NL, Tettelin H, Davidsen TM, Beanan MJ, Deboy RT, Daugherty SC, Brinkac LM, Madupu R, Dodson RJ, Khouri HM, Lee KH, Carty HA, Scanlan D, Heinzen RA, Thompson HA, Samuel JE, Fraser CM, Heidelberg JF: Complete genome sequence of the Q-fever pathogen Coxiella burnetii. Proc Nat Acad Sci USA 2003 100:5455-5460

14. Kawashima T, Amano N, Koike H, Makino S, Higuchi S, KawashimaOhya Y, Watanabe K, Yamazaki M, Kanehori K, Kawamoto T, Nunoshiba T, Yamamoto Y, Aramaki H, Makino K, Suzuki M: Archaeal adaptation to higher temperatures revealed by genomic sequence of Thermoplasma volcanium. Proc Natl Aca Sci USA 2000, 97:|4257-|4262.

I5. Nandi S, Mehra N, Lynn A, Bhattacharya A: Comparison of theoretical proteome: Identification of $C O G s$ with conserved and variable PI with the multimodal PI distribution. BMC Genomics 2005, 6:1 16

16. Baldauf SL: A Search for the Origins of Animals and Fungi: Comparing and Combining Molecular Data. Am Nat 1999 I 54(S4):SI78-SI88.

17. Baldauf SL, Roger AJ, Wenk-Siefert I, Doolittle WF: A kingdomlevel phylogeny of eukaryotes based on combined protein data. Science 2000, 290:972-977.

18. Lanyi JK: Salt-dependent properties of proteins from extremely halophilic bacteria. Bacteriol Rev 1974, 38:272-290.

19. Bonnete F, Madern D, Zaccai G: Stability against denaturation mechanisms in halophilic malate dehydrogenase "adapt" to solvent conditions. J Mol Biol 1994, 244:436-447.

20. Ng WV, Kennedy S, Mahairas GG, Berquist B, Pan M, Shukla HD, Lasky SR, Baliga N, Thorsson V, Sbrogna J, Swartzell S, Weir D, Hall J, Dahl TA, Welti R, Goo YA, Leithauser B, Keller K, Cruz R, Danson MJ, Hough DW, Maddocks DG, Jablonski PE, Krebs MP, Angevine CM, Dale H, Isenbarger TA, Peck RF, Pohlschroder M, Spudich JL,
Jung K, Alam M, Freitas T, Hou S, Daniels Cl, Dennis PP, Omer AD, Ebhardt H, Lowe TM, Liang P, Riley M, Hood L, DasSarma S: Genome Sequence of Halobacterium Species NRC-I. Proc Nat Acad Sci USA 2000, 97:12176-12181.

21. Karlin S, Brocchieri L, Trent J, Blaisdell BE, Mrazek J: Heterogeneity of genome and proteome content in Bacteria, Archaea, and Eukaryotes. Theor Popul Biol 2002, 6 I:367-390.

22. Fukuchi S, Yoshimune K, Wakayama M, Moriguchi M, Nishikawa K: Unique amino acid composition of proteins in halophilic bacteria. I Mol Biol 2003, 327:347-357.

23. Rao JK, Argos P: Structural stability of halophilic proteins. Biochemistry 198I, 20:6536-6543.

24. Dym O, Mevarech JL, Sussman L: Structural features that stabilize halophilic malate dehydrogenase from an archaebacterium. Science 1995, 267:1334-1346.

25. Eisenberg $\mathrm{H}$ : Life in unusual environments: progress in understanding the structure and function of enzymes from extreme halophilic bacteria. Arch Biochem Biophys 1995, 3 I 8: I-5.

26. Madern D, Zaccai G: Stabilisation of halophilic malate dehydrogenase from Haloarcula marismortui by divalent cations effects of temperature, water isotope, cofactor and $\mathrm{pH}$. Eur J Biochem 1997, 249:607-6I I.

27. Elcock $\mathrm{AH}, \mathrm{McCammon} \mathrm{JA}$ : Electrostatic contributions to the stability of halophilic proteins. I Mol Biol I998, 280:73|-748 [http://www.genome.org/cgi/external ref?access num $=10.1006 /$ jmbi. 1998.1904\&link type=DOI]

28. Oren A, Mana L: Amino acid composition of bulk protein and salt relationships of selected enzymes of Salinibacter ruber, an extremely halophilic Bacterium. Extremophiles 2002 , 6:217-223.

29. Teysseire N, Boudier JA, Raoult D: Rickettsia conorii entry into Vero cells. Infect Immun 1995, 63:366-374.

30. Douglas S, Zauner S, Fraunholz M, Beaton M, Penny S, Deng LT, Wu $X$, Reith M, Cavalier-Smith T, Maier UG: The highly reduced genome of an enslaved algal nucleus. Nature 200I, $410: 1091-1096$

31. Moran NA: Genome reduction in bacterial pathogens. Cell 2002, I 08:583-586.

32. Shigenobu S, Watanabe H, Hattori M, Sakaki Y, Ishikawa H: Genome sequence of the endocellular bacterial symbiont of aphids Buchnera sp. APS. Nature 2000, 407:8I-86.

33. Akman L, Yamashita A, Watanabe H, Oshima K, Shiba T, Hattori M, Aksoy S: Genome sequence of the endocellular obligate symbiont of tsetse flies, Wigglesworthia glossinidia. Nat Genet 2002, 32:402-407.

34. Moran NA: Tracing the evolution of gene loss in obligate symbionts. Curr Opin Microbiol 2003, 6:512-518.

35. Muto A, Osawa S: The guanine and cytosine content of genomic DNA and bacterial evolution. Proc Natl Acad Sci USA 1987, 84: 166-169.

36. Itoh T, Martin W, Nei M: Acceleration of genomic evolution caused by enhanced mutation rate in endocellular symbionts. Proc Natl Acad Sci USA 2002, 99: I 2944-12948.

37. Moran NA: Accelerated evolution and Muller's rachet in endosymbiotic bacteria. Proc Nat Acad Sci USA 1996, 93:2873-2878.

38. Andersson SGE, Kurland CG: Reductive evolution of resident genomes. Trends Microbiol 1998, 6:263-268.

39. Lambert JL, Moran NA: Deleterious mutations destabilize ribosomal RNA of endosymbionts. Proc Natl Acad Sci USA 1998, 95:4458-4462.

40. Clark MA, Moran NA, Baumann P: Sequence evolution in bacterial endosymbionts having extreme base composition. Mol Biol Evol 1999, 16:1586-1598.

4I. Wernegreen JJ, Moran NA: Evidence for genetic drift in endosymbionts (Buchnera): analyses of protein-coding genes. Mo Biol Evol 1999, 16:83-97.

42. Funk DJ, Wernegreen J, Moran NA: Intraspecific variation in symbiont genomes: bottlenecks and the aphid Buchnera association. Genetics 200I, I 57:477-489.

43. Moran NA, Mira A: The process of genome shrinkage in the obligate symbiont, Buchnera aphidicola. Genome Biol 2001, 2: $1-0054$

44. van Ham RCHJ, Kamerbeek J, Palacios C, Rausell C, Abascal F, Bastolla U, Fernández JM, Jiménez L, Postigo M, Silva FJ, Tamames J, Viguera E, Latorre A, Valencia A, Morán F, Moya A: Reductive genome 
evolution in Buchnera aphidicola. Proc Nat Acad Sci USA 2003, 100:58I-586.

45. Woolfit M, Bromham L: Increased rates of sequence evolution in endosymbiotic bacteria and fungi with small effective population sizes. Mol Biol Evol 2003, 20:1545-I555.

46. Wu M, Sun LV, Vamathevan J, Riegler M, Deboy R, Brownlie JC, McGraw EA, Martin W, Esser C, Ahmadinejad N, Wiegand C, Madupu R, Beanan MJ, Brinkac LM, Daugherty SC, Durkin AS, Kolonay JF, Nelson WC, Mohamoud Y, Lee P, Berry K, Young MB, Utterback T, Weidman J, Nierman WC, Paulsen IT, Nelson KE, Tettelin H, O'Neill SL, Eisen JA: Phylogenomics of the reproductive parasite Wolbachia pipientis wMel: a streamlined genome overrun by mobile genetic elements. PLOS Biol 2004, 2:0327-034I.

47. Moran NA, Wernegreen J]: Are mutualism and parasitism irreversible evolutionary alternatives for endosymbiotic bacteria? Insights from molecular phylogenetics and genomics. Trends Ecol Evol 2000, 1 5:32I-326.

48. Tamas I, Klasson L, Canbäck B, Näslund K, Eriksson AS, Sandström J, Wernegreen J, Moran NA, Andersson SGE: 50 million years of genomic stasis in endosymbiotic bacteria. Science 2002, 296:2376-2379.

49. Klasson L, Andersson SG: Evolution of minimal-gene-sets in host-dependent bacteria. Trends Microbiol 2004, I 2:37-43.

50. The UniProt Consortium : The Universal Protein Resource (UniProt). Nucleic Acids Res 2007, 35:D I 93-D 197.

5I. Guo T, Hua S, Ji X, Sun Z: DBSubLoc: database of protein subcellular localization. Nucleic Acids Res 2004, 32:DI22-124.

52. Bjellqvist B, Hughes G], Pasquali Ch, Paquet N, Ravier F, Sanchez JCh, Frutiger S, Hochstrasser D: The focusing positions of polypeptides in immobilized $\mathrm{pH}$ gradients can be predicted from their amino acid sequences. Electrophoresis 1993, 14:1023-1031.

53. Bjellqvist B, Basse B, Olsen E, Celis JE: Reference points for comparisons of two-dimensional maps of proteins from different human cell types defined in a $\mathrm{pH}$ scale where isoelectric points correlate with polypeptide compositions. Electrophoresis 1994, 15:529-539.

54. Gasteiger E, Hoogland C, Gattiker A, Duvaud S, Wilkins MR, Appel $R D$, Bairoch A: Protein Identification and Analysis Tools on the ExPASy Server. In The Proteomics Protocols Handbook Volume II2. Edited by: Walker JM. Humana Press. Full text - Copyright Humana Press; 2005:53I-552.

55. Felsenstein J: PHYLIP (Phylogeny Inference Package) version 3.6. In Distributed by the author. Department of Genome Sciences University of Washington, Seattle; 2004.

56. Sanjuan R, Wrobel B: Weighted least-squares likelihood ratio test for branch testing in phylogenies reconstructed from distance measures. Syst Biol 2005, 54:2 18-229.

57. Uchiyama I: MBGD: microbial genome database for comparative analysis. Nucleic Acids Res 2003, 31 : 58-62.

58. Benjamini $Y$, Hochberg $Y$ : Controlling the false discovery rate: a practical and powerful approach to multiple testing. J Roy Statist Soc Ser B 1995, 57:289-300.

\section{Publish with Biomed Central and every scientist can read your work free of charge}

"BioMed Central will be the most significant development for disseminating the results of biomedical research in our lifetime. "

Sir Paul Nurse, Cancer Research UK

Your research papers will be:

- available free of charge to the entire biomedical community

- peer reviewed and published immediately upon acceptance

- cited in PubMed and archived on PubMed Central

- yours - you keep the copyright
BiolMedcentral 